\title{
Patterns of Treatment Sequences in Chemotherapy and Targeted Biologics for Metastatic Colorectal Cancer: Findings from a Large Community-Based Cohort of Elderly Patients
}

\author{
Rohan C. Parikh ${ }^{1}$ Xianglin L. Du ${ }^{1,2} \cdot$ Robert O. Morgan' ${ }^{1}$ David R. Lairson ${ }^{1}$
}

Published online: 19 January 2016

(c) The Author(s) 2016. This article is published with open access at Springerlink.com

\begin{abstract}
Background Over the last decade, multiple chemotherapies/targeted biologics have been approved for metastatic colorectal cancer (mCRC). However, evidence is limited with regards to the array of treatments received by mCRC patients.

Objective This study examines treatment sequences (firstto third-line chemotherapy/targeted biologics) and the factors associated with first-line targeted biologics and common treatment sequences for elderly mCRC patients treated in a community setting.

Methods A retrospective cohort study was conducted in mCRC patients diagnosed from January 2004 through December 2009 using the Surveillance, Epidemiology and End Results Medicare-linked database. The treatment sequences administered to elderly mCRC patients were empirically identified.

Results Of 4418 mCRC patients who received treatment, 1370 (31\%) received first, second, and third line; 1164 (26\%) received first and second line; and 1884 (43\%) received only first line. The most common first line of
\end{abstract}

Electronic supplementary material The online version of this article (doi:10.1007/s40801-015-0059-9) contains supplementary material, which is available to authorized users.

Rohan C. Parikh

rohanparikh23@gmail.com; rohan.c.parikh@uth.tmc.edu

1 Division of Management, Policy and Community Health, School of Public Health, University of Texas Health Science Center at Houston, 1200 Pressler Dr, RAS-E929, Houston, TX 77030, USA

2 Division of Epidemiology, Human Genetics and Environmental Sciences, School of Public Health, University of Texas Health Science Center at Houston, Houston, TX, USA treatment for mCRC patients was 5-fluorouracil/leucovorin + oxaliplatin (FOLFOX) + bevacizumab $(23 \%)$ and FOLFOX (23\%). 5-fluorouracil/leucovorin + irinotecan (FOLFIRI)-based regimens were commonly (22\%) administered in second line. The most common treatment sequence was first-line oxaliplatin or irinotecan followed by second-line oxaliplatin or irinotecan + bevacizumab followed by a third-line targeted biologic. Of patients who received first-line therapy, $47 \%$ also received a targeted biologic, and the factors associated were age, comorbidity score, cancer site, geographic location, and year of diagnosis. Conclusion Elderly mCRC patients receive a multitude of treatments in various sequences. Further exploration of the comparative effectiveness of treatment sequences may yield important information for improving $\mathrm{mCRC}$ survival.

\section{Key Points}

Elderly metastatic colorectal cancer patients received treatment sequences with multiple drugs administered across various lines of treatment.

Oxaliplatin- or irinotecan-based regimens were the most common chemotherapies, bevacizumab was the most common targeted biologic, and the most common treatment sequence was first-line oxaliplatin or irinotecan followed by second-line oxaliplatin or irinotecan + bevacizumab followed by a third-line targeted biologic.

Future research evaluating the comparative effectiveness and cost effectiveness of treatment lines and sequences for elderly patients with metastatic colorectal cancer should be conducted. 


\section{Introduction}

Colorectal cancer (CRC) currently ranks third among the most common cancers and cancer deaths in the USA [1-3]. It is estimated there will be about 132,700 new cases of CRC and nearly 49,700 deaths because of CRC in 2015 in the USA $[1,3]$. A majority of cases $(60 \%)$ and deaths (70 \%) occur in those aged $\geq 65$ years. For males between the ages of 40 and 79 years and females aged $\geq 80$ years, CRC is the second leading cause of death [3]. As compared with younger CRC patients, elderly CRC patients have a lower survival rate primarily because of the stage at diagnosis. Moreover, the management of the disease among elderly patients is also poor. Overall, one in four patients has the metastatic form of the disease at diagnosis, and nearly half of CRC patients may develop metastasis during progression of the disease. Metastatic colorectal cancer (mCRC) has a poor prognosis, with an overall survival rate of $5-13 \%$ at 5 years $[4,5]$, and the cost of treating metastatic disease is twice as high as the cost of cases without metastasis [6].

Until 2004, 5-Fluorouracil, leucovorin (5-FU/LV) had been the standard therapy for mCRC patients, with an estimated median overall survival of 10-14 months. Oxaliplatin and irinotecan in combination with 5-FU/LV, i.e., FOLFOX (5-FU/LV + oxaliplatin) and FOLFIRI (5FU/LV + irinotecan), respectively, have been commonly prescribed to mCRC patients since 2004 [7]. Targeted biologics such as bevacizumab and cetuximab were approved for treating mCRC patients in 2004, which was followed by the approval of panitumumab in 2006. These clinically proven therapies are current standard treatments that can be administered either as monotherapy or as a combination to form a treatment line. With an array of chemotherapy/targeted therapy options available for mCRC patients, multiple lines of treatment could be administered to a patient as needed during the course of their treatment and thereby form a treatment sequence, where each sequence comprises multiple lines of treatments [7-9]. Currently, there is a lack of standard sequence of chemotherapy and targeted biologics recommended for mCRC patients [8-13]. In the absence of evidence-based guidelines for sequencing therapy, the decision regarding first-line treatment has been generally based on patient factors and preferences while subsequent treatments (after progression) are based on the treatment previously received [14].

Recommendations have been made for healthy elderly patients to be treated with chemotherapy and targeted biologic combinations similar to those administered to younger patients [15]. Specifically, irinotecan (e.g., FOLFIRI)- or oxaliplatin (e.g. FOLFOX)-based regimens with or without bevacizumab for first- and second-line treatment may be the treatment of choice [16-18]. No specific recommendations have been made for the third line of treatment, but targeted biologics have been used in one study and are currently being evaluated in ongoing clinical trials $[11,19,20]$. Although multiple treatment options may be available for mCRC patients, elderly patients have been observed to frequently receive suboptimal treatment, and only a subgroup of elderly patients may receive exhaustive treatment management similar to that received by younger patients [21-25]. Thus, an understanding of the demographic and clinical factors associated with various treatments received by elderly mCRC patients is essential. Moreover, evidence is limited on the current usage of treatment sequences among elderly mCRC patients treated in a non-experimental (community-based) setting, especially with regards to targeted biologics; assessing realworld utilization of treatment sequences may guide in optimizing the adequate sequential use of targeted biologics in routine practice and in-turn judicious use of healthcare resources. Thus, the objective of the study was to describe treatment sequences (first- to third-line chemotherapy and targeted biologics) and the factors associated with the receipt of targeted biologics at first-line and treatment sequences for elderly mCRC patients in community-based settings.

\section{Methods}

\subsection{Data Source}

The National Cancer Institute governs the Surveillance, Epidemiology and End Results (SEER) program under which participating regions provide cancer registry data that includes information on patient demographics, socioeconomic variables, stage at diagnosis, tumor site, tumor characteristics, and initial treatment after diagnosis. After the expansion of the SEER program in 2000, the 16 participating registries (i.e., San Francisco/Oakland, Detroit, Seattle, Atlanta, Rural Georgia, Los Angeles, San JoseMonterey area, Greater California, Connecticut, Iowa, New Mexico, Utah, Hawaii, Kentucky, Louisiana, and New Jersey) represent nearly $28 \%$ of the US population, and SEER records $98 \%$ of the cancer-diagnosed cases in these regions [26, 27]. These data have been used for numerous cancer epidemiology and chemotherapy utilization studies; validity and completeness of the database has also been shown in previous studies [28-30]. The SEER-Medicare data linked cancer patients aged $\geq 65$ years from the SEER program to their administrative claims from the Medicare program, which insures individuals aged $>65$ years in the 
USA [26]. Medicare data includes healthcare utilization information for inpatient, outpatient, professional (provider), skilled nursing facility, hospice, and devices and medical equipment.

\subsection{Study Population}

Patients diagnosed with $\mathrm{mCRC}$ at $\geq 65$ years from January 2004 to December 2009 were included. Targeted therapies such as bevacizumab and cetuximab became available for mCRC patients in 2004; hence, analysis was restricted to patients diagnosed after 2004. We used an American Joint Cancer Committee (AJCC) criterion to characterize metastatic disease, and patients with AJCC stage IV were included. Patients who were ascertained as mCRC through autopsy/ death certificate were excluded, as patients had already died before receiving any treatment. Also, patients who died within 30 days of diagnosis were excluded as they were unlikely to have received treatment sequences [14, 31, 32]. For the completeness of information on treatment sequences in Medicare claims, patients were required to be enrolled in both Medicare parts A and B without any Health Maintenance Organization (HMO) enrollment from the time of diagnosis to death or end of study. Similar inclusion/exclusion criteria have been used in previous studies [31, 33-36].

\subsection{Treatment Identification}

We identified systemic chemotherapy and targeted biologics currently approved by the US FDA for treatment of $\mathrm{mCRC}$ patients and recommended by the National Comprehensive Cancer Network [7-9], i.e., 5-florouracil, irinotecan, oxaliplatin, bevacizumab, cetuximab, and panitumumab. Aflibercept, although approved in 2012, was not included in this study, as Medicare claims were only available until 2010. Chemotherapeutic and targeted biologics agents could be given either as monotherapy or as a combination therapy to form a 'line of treatment'. We identified the first three lines of treatment administered to mCRC patients and used Healthcare Common Procedural Coding System (HCPCS) codes from the Medicare outpatient and physician files to identify chemotherapy or targeted biologics. HCPCS codes used were 5-florouracilJ9190; irinotecan-J9206; leucovorin-J0640, J0641; oxaliplatin_J9263, C9205; bevacizumab_J9035, C9214, S0116; cetuximab-J9055, C9215, and panitumumabJ9303, C9235.

\subsection{Line and Sequence Identification}

A data-driven 'line of treatment' approach was used to identify the treatment sequences. Start of a line of treatment was determined based on the date of the first claim for the drug.
Additionally, for the drug to be considered as a line of treatment, it was required to be re-administered within 35 days $(28+7$ additional days). A combination regimen was defined when an additional drug was administered within 28 days of the first drug claim and was re-administered within 35 days $(28+7$ additional days). End of a line of treatment was defined as (1) a line continues until the end of the study; (2) no drug is administered within 90 days, or (3) a previous line of treatment is interrupted by a new line of treatment [37]. This process was conducted three times to identify three treatment lines. Similar methodology has been used by previous treatment pattern studies [11, 38, 39]. For patients receiving at least two lines of treatments, first- to third-line treatments were combined to define treatment sequences. Finally, we only included patients for whom the gap between treatment lines (first to second line and second to third line) was less than 1 year.

\subsection{Patient and Tumor Characteristics}

SEER data records demographic information such as age, race, sex, marital status, year of diagnosis, and geographic location at the time of diagnosis. The variable "percent below poverty line at zip code level" obtained from the US Census Data was used as a proxy for patient's socio-economic (poverty) status. The poverty variable was then categorized into quartiles to differentiate individuals living in areas with higher versus lower rates of poverty. Tumor stage, grade and site of cancer (i.e., colon or rectal) were obtained from SEER data. A Charlson Comorbidity Index (CCI) was computed with inpatient, outpatient, and physician claims from 1 year prior to the month of diagnosis using non-cancer comorbid conditions initially identified by Charlson et al. [40-42] to affect overall morbidity and mortality. Metastases type was identified using inpatient, outpatient, and physician claims within 3 months after diagnosis based on the algorithm used by Chawla et al. [43]. International Classification of Diseases, Ninth Revision, Clinical Modification (ICD-9-CM) codes were used to identify metastases [Table 1 in the Electronic Supplementary Marerial (ESM)], and patients were considered to have metastases if they had at least one inpatient claim or two outpatient/provider claims on separate days [29, 43, 44].

\subsection{Statistical Analyses}

Descriptive statistics (mean, standard deviation, and median time) for each of the treatments (monotherapy or combination) in first, second, and third line, as well as treatment sequences, were calculated. We computed descriptive statistics for patients receiving targeted biologics at first line, and used a logistic regression analysis to assess factors associated with the receipt of targeted biologics in first line. Factors associated with the receipt of commonly administered targeted biologics-based treatment 
Table 1 Characteristics of metastatic colorectal cancer patients and patients

\begin{tabular}{lll}
\hline Characteristics & $\begin{array}{l}\text { All MCRC pts } \\
(n=9819)\end{array}$ & $\begin{array}{l}\text { Pts with mCRC } \\
\text { who received either } \\
\text { CTX or targeted } \\
\text { therapy }(n=4418)\end{array}$ \\
&
\end{tabular}

Age (years)

65-69

70-74

75-79

$80-84$

$\geq 85$

Race/ethnicity

Caucasians

African Americans

Other

Sex

Male

Female

Marital status

Married

Unmarried

Unknown

Tumor grade

Well/moderately differentiated

Poorly/undifferentiated

Unknown

Comorbidity scores

0

1

2

$\geq 3$

Metastasis

Liver

Lung

Abdomen

Other

Unknown

Cancer site

Colon

Rectal

SES (poverty)

1st (low SES)

2nd

3rd

4th (high SES)

Region

Midwest

North east

South

West

Urban/rural

Less urban/rural

Urban

Metro
2098 (21.4)

2092 (21.3)

2083 (21.2)

1851 (18.9)

1695 (17.3)

7924 (80.7)

1095 (11.2)

800 (8.2)

4720 (48.1)

5099 (51.9)

$4714(48.0)$

336 (3.4)

$5112(52.1)$

2438 (24.8)

2269 (23.1)

4712 (48.0)

2677 (27.3)

1227 (12.5)

1203 (12.3)

6234 (63.5)

1692 (17.2)

1953 (19.9)

1618 (16.5)

1969 (20.1)

7559 (77.0)

$2260(23.0)$

2454 (25.0)

2404 (24.5)

2502 (25.5)

2459 (25.0)

1284 (13.1)

2212 (22.5)

2275 (23.2)

4048 (41.2)

1333 (30.2)

1190 (26.9)

1022 (23.1)

627 (14.2)

246 (5.6)

3693 (83.6)

$382(8.7)$

343 (7.8)

2318 (52.5)

$2100(47.5)$

2571 (58.2)

1718 (38.9)

129 (2.9)

2554 (57.8)

1148 (26.0)

$716(16.2)$

2357 (53.4)

1280 (29.0)

430 (9.7)

$351(7.9)$

2969 (67.2)

$730(16.5)$

$873(19.8)$

$696(15.8)$

747 (16.9)

3276 (74.2)

1142 (25.9)

$989(22.4)$

$1070(24.2)$

1139 (25.8)

1220 (27.6)

$566(12.8)$

1000 (22.6)

1035 (23.4)

1817 (41.1)

$\begin{array}{cc}1097(11.2) & 477(10.8) \\ 588(6.0) & 268(6.1) \\ 8132(82.8) & 3672(83.1)\end{array}$

4769 (48.6)
Table 1 continued

\begin{tabular}{lll}
\hline Characteristics & $\begin{array}{l}\text { All MCRC pts } \\
(n=9819)\end{array}$ & $\begin{array}{l}\text { Pts with mCRC } \\
\text { who received either } \\
\text { CTX or targeted } \\
\text { therapy }(n=4418)\end{array}$ \\
\hline $\begin{array}{l}\text { Year of diagnosis } \\
2004\end{array}$ & $1588(16.2)$ & $731(16.6)$ \\
2005 & $1510(15.4)$ & $696(15.8)$ \\
2006 & $1811(18.4)$ & $780(17.7)$ \\
2007 & $1668(17.0)$ & $722(16.3)$ \\
2008 & $1650(16.8)$ & $752(17.0)$ \\
2009 & $1592(16.2)$ & $737(16.7)$ \\
\hline
\end{tabular}

Data are presented as $n(\%)$

CTX chemotherapy, $m C R C$ metastatic colorectal cancer, pts patients, SES socio-economic status

sequences were assessed using univariate chi-squared statistic and multinomial logistic regression. In contrast to conventional logistic regression, multinomial logistic regression allowed the use of dependent variables with more than two categories and thereby enabled us to examine any association between multiple treatment sequences and patient/tumor characteristics [45-47]. All analyses were conducted using SAS version 9.3 , and statistical significance was determined at $\alpha=0.05$.

\section{Results}

Of the 9819 patients diagnosed with mCRC from January 2004 to December 2009 who met other inclusion criteria (Fig. 1 in the ESM); $5192(53 \%)$ did not receive treatment, $4418(45 \%)$ received treatment, and $209(2 \%)$ were excluded, as the gap between first to second line or second to third line was more than 1 year. The baseline characteristics for all mCRC patients and patients who received treatment are shown in Table 1. Overall, the sample comprised $81 \%$ Caucasians, $52 \%$ females, $83 \%$ living in a metropolitan area, $77 \%$ with metastatic colon cancer, and $23 \%$ with metastatic rectal cancer (Table 1 ). A majority of patients had a liver metastasis $(63 \%)$, followed by abdomen (20\%) and lung (17\%). We were not able to identify the type of metastases in $20 \%$ of patients (Table 1) even though they were indicated as metastatic (AJCC stage IV) in SEER. Patients who received treatment were mostly diagnosed before the age of 80 years $(80 \%)$ and had a comorbidity score of 0 or $1(82 \%)$.

\subsection{Treatment Lines and Sequences}

Of the 4418 patients who received treatment, 1370 (31\%) received first-, second-, and third-line treatment, 1164 (26\%) received first and second-line treatment, and 1884 (43\%) received only first-line treatment. Table 2 shows the top ten 
treatment regimens for first-, second-, and third-line treatment, along with duration of therapy. The most common first-line treatments were FOLFOX (oxaliplatin based) + bevacizumab $(23 \%)$, FOLFOX [oxaliplatin based $(23 \%)$ ] alone and 5-FU + leucovorin (12\%) administered for a median duration of 188, 124, and 97 days, respectively (Table 2). In second-line treatment, FOLFOX (oxaliplatin based) + bevacizumab (18\%) was the most common regimen, followed by FOLFIRI (irinotecan based) + bevacizumab $(14 \%)$ and
FOLFIRI (irinotecan based) alone ( $8 \%$ ). The median duration for FOLFOX (oxaliplatin based) + bevacizumab, FOLFIRI (irinotecan based) + bevacizumab, and FOLFIRI (irinotecan based) alone was observed to be 156, 155, and 111 days, respectively (Table 2). The most common regimens administered in third-line treatment (Table 2) were cetuximab + irinotecan $(15 \%)$, FOLFIRI (irinotecan based) + bevacizumab $(13 \%)$, and FOLFOX (oxaliplatin based) + bevacizumab $(8 \%)$.
Table 2 Treatment regimens and duration for metastatic colorectal cancer patients by line of therapy

\begin{tabular}{|c|c|c|c|c|}
\hline \multirow[t]{2}{*}{ Treatment line and regimens } & \multirow[t]{2}{*}{ Patients, $n(\%)$} & \multicolumn{3}{|c|}{ Duration (days) } \\
\hline & & Mean & SD & Median \\
\hline First line & $N=4418$ & & & \\
\hline FOLFOX + bevacizumab & $1026(23.2)$ & 197.5 & 115.5 & 188 \\
\hline FOLFOX & $1003(22.7)$ & 139.1 & 94.4 & 124 \\
\hline FU/LV & $510(11.5)$ & 130.9 & 111.1 & 97 \\
\hline Oxaliplatin & $325(7.4)$ & 126.9 & 92.3 & 104 \\
\hline FU/LV + bevacizumab & $218(4.9)$ & 180.2 & 153.2 & 132 \\
\hline Oxaliplatin + bevacizumab & $216(4.9)$ & 186.9 & 114.3 & 167 \\
\hline FOLFIRI + bevacizumab & $194(4.4)$ & 208.7 & 158.5 & 177 \\
\hline FOLFIRI & $183(4.1)$ & 149.2 & 114.0 & 136 \\
\hline Bevacizumab & $151(3.4)$ & 186.3 & 144.0 & 145 \\
\hline FU & $150(3.4)$ & 84.2 & 57.3 & 67 \\
\hline Others & $442(10.0)$ & 135.8 & 100.0 & 111 \\
\hline Second line & $N=2534$ & & & \\
\hline FOLFOX + bevacizumab & 449 (17.7) & 183.3 & 136.5 & 156 \\
\hline FOLFIRI + bevacizumab & $353(13.9)$ & 198.0 & 157.2 & 155 \\
\hline FOLFIRI & $202(8.0)$ & 128.0 & 83.5 & 111 \\
\hline Irinotecan & $192(7.6)$ & 126.4 & 93.0 & 97 \\
\hline FU/LV + bevacizumab & $175(6.9)$ & 181.1 & 143.1 & 139 \\
\hline FOLFOX & $157(6.2)$ & 132.6 & 74.3 & 120 \\
\hline Cetuximab + irinotecan & $139(5.5)$ & 155.5 & 98.8 & 135 \\
\hline Oxaliplatin + bevacizumab & $127(5.0)$ & 164.3 & 113.9 & 128 \\
\hline Bevacizumab & $116(4.6)$ & 194.1 & 207.3 & 133 \\
\hline FU/LV & $98(3.9)$ & 144.9 & 128.8 & 118 \\
\hline Others & $526(20.8)$ & 138.3 & 104.7 & 117 \\
\hline Third line & $N=1370$ & & & \\
\hline Cetuximab + irinotecan & $207(15.1)$ & 152.0 & 124.7 & 125 \\
\hline FOLFIRI + bevacizumab & $184(13.4)$ & 195.0 & 158.8 & 153 \\
\hline FOLFOX + bevacizumab & $104(7.6)$ & 162.1 & 104.6 & 138 \\
\hline FOLFIRI & $91(6.6)$ & 119.7 & 105.3 & 96 \\
\hline Irinotecan & $82(6.0)$ & 123.0 & 89.2 & 89 \\
\hline Cetuximab & $78(5.7)$ & 131.5 & 137.3 & 101 \\
\hline FU/LV + bevacizumab & $75(5.5)$ & 199.1 & 201.3 & 132 \\
\hline Bevacizumab & $71(5.2)$ & 215.2 & 180.1 & 166 \\
\hline Bevacizumab + irinotecan & $57(4.2)$ & 167.2 & 109.9 & 134 \\
\hline FOLFOX & $51(3.7)$ & 120.8 & 71.5 & 98 \\
\hline Others & $370(30.7)$ & 135.9 & 112 & 110 \\
\hline
\end{tabular}

FU 5-fluorouracil, FOLFOX 5-FU + LV+ oxaliplatin, FOLFIRI 5-FU + LV + irinotecan, $L V$ leucovorin, $S D$ standard deviation 


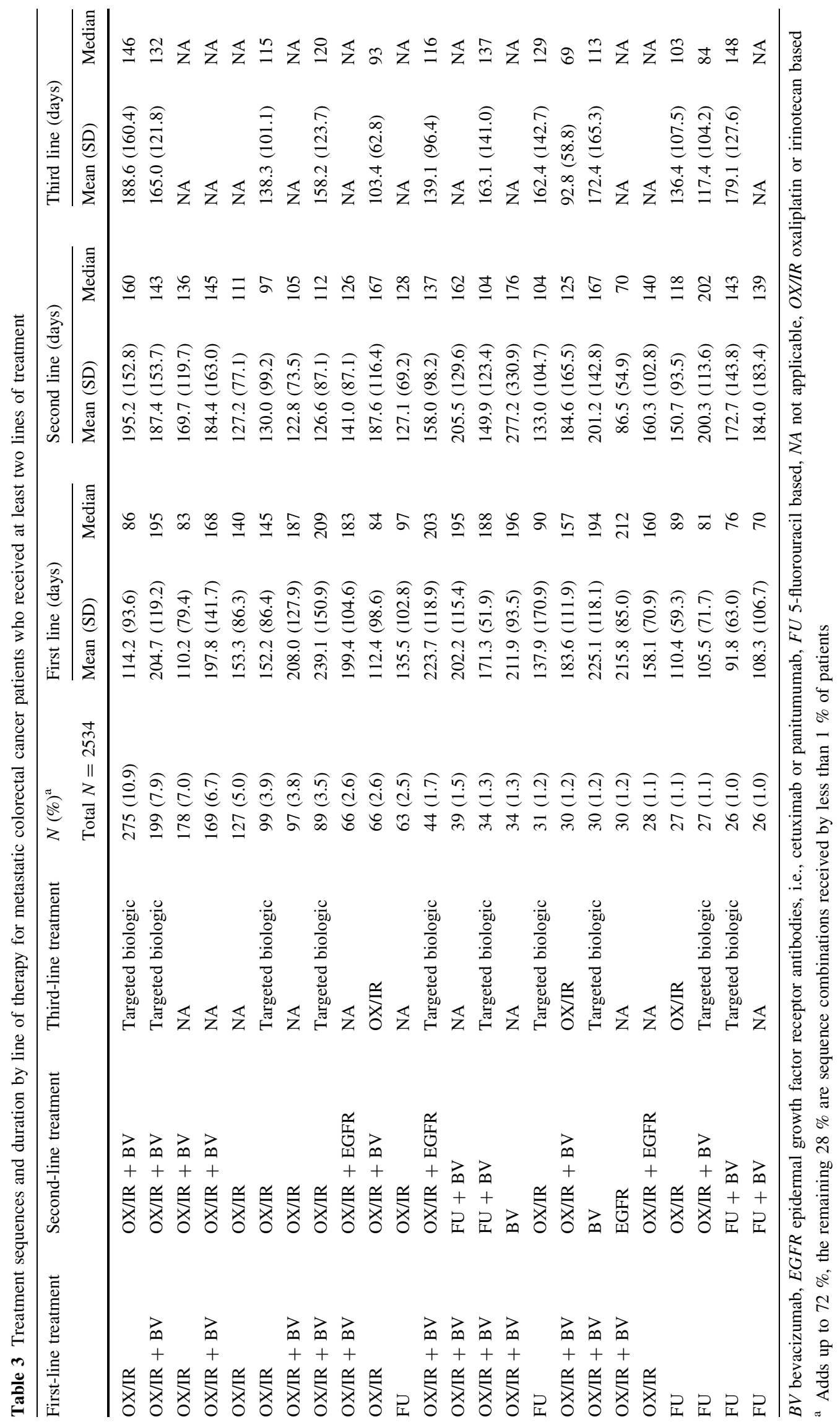


Table 4 Characteristics of metastatic colorectal cancer patients receiving targeted biologic in first line and multivariable regression for factors associated with the receipt of targeted biologics

\begin{tabular}{|c|c|c|c|}
\hline Characteristics & $\begin{array}{l}\text { mCRC patients who } \\
\text { received first-line } \\
\text { treatment, } \\
N(\text { column \%) }\end{array}$ & $\begin{array}{l}\text { mCRC patients who } \\
\text { received first-line with } \\
\text { targeted biologic, } \\
N(\text { row \%) }\end{array}$ & $\begin{array}{l}\text { Receipt of first-line } \\
\text { targeted biologic, } \\
\text { multivariable } \\
\text { OR }(95 \% \text { CI })\end{array}$ \\
\hline Total & $N=4418$ & $N=2077$ & \\
\hline \multicolumn{4}{|l|}{ Age (years) } \\
\hline $65-69$ & $1333(30.2)$ & $664(49.8)$ & Referent \\
\hline $70-74$ & $1190(26.9)$ & $555(46.6)$ & $0.88(0.74-1.04)$ \\
\hline $75-79$ & $1022(23.1)$ & $451(44.1)$ & $0.77(0.64-0.91)^{*}$ \\
\hline $80-84$ & $627(14.2)$ & $280(44.7)$ & $0.78(0.64-0.96)^{*}$ \\
\hline $85+$ & $246(5.6)$ & $127(51.6)$ & $0.99(0.74-1.33)$ \\
\hline \multicolumn{4}{|l|}{ Race/ethnicity } \\
\hline Caucasians & 3693 (83.6) & $1732(46.9)$ & Referent \\
\hline African Americans & $382(8.7)$ & $181(47.4)$ & $0.94(0.74-1.20)$ \\
\hline Others & $343(7.8)$ & $164(47.8)$ & $1.05(0.82-1.34)$ \\
\hline \multicolumn{4}{|l|}{ Sex } \\
\hline Male & $2318(52.5)$ & $992(42.8)$ & Referent \\
\hline Female & $2100(47.5)$ & $1085(51.7)$ & $0.97(0.85-1.10)$ \\
\hline \multicolumn{4}{|l|}{ Marital status } \\
\hline Married & $2571(58.2)$ & $1190(46.3)$ & Referent \\
\hline Unmarried & $1718(38.9)$ & $828(48.2)$ & $1.09(0.96-1.25)$ \\
\hline Unknown & $129(2.9)$ & $59(45.7)$ & $1.07(0.73-1.56)$ \\
\hline \multicolumn{4}{|l|}{ Tumor grade } \\
\hline Well/moderately differentiated & $2554(57.8)$ & $1196(46.8)$ & Referent \\
\hline Poorly/undifferentiated & $1148(26.0)$ & $542(47.2)$ & $1.04(0.90-1.21)$ \\
\hline Unknown & $716(16.2)$ & $339(47.3)$ & $1.03(0.86-1.23)$ \\
\hline \multicolumn{4}{|l|}{ Comorbidity Scores } \\
\hline 0 & $2357(53.4)$ & $1122(47.6)$ & Referent \\
\hline 1 & $1280(29.0)$ & $585(45.7)$ & $0.85(0.73-0.98)^{*}$ \\
\hline 2 & $430(9.7)$ & $211(49.1)$ & $1.00(0.80-1.25)$ \\
\hline$\geq 3$ & $351(7.9)$ & $159(45.3)$ & $0.80(0.63-1.01)$ \\
\hline \multicolumn{4}{|l|}{ Metastasis } \\
\hline Liver & $2969(67.2)$ & 1476 (49.7) & $1.14(0.93-1.38)$ \\
\hline Lung & $730(16.5)$ & $358(49.0)$ & $1.06(0.89-1.26)$ \\
\hline Abdomen & $873(19.8)$ & $423(48.5)$ & $1.00(0.83-1.20)$ \\
\hline Other & $696(15.8)$ & $304(43.7)$ & $0.77(0.64-0.92)^{*}$ \\
\hline Unknown & $747(16.9)$ & $283(37.9)$ & $0.68(0.52-0.88)^{*}$ \\
\hline \multicolumn{4}{|l|}{ Cancer site } \\
\hline Colon & $3276(74.2)$ & $1618(49.4)$ & Referent \\
\hline Rectal & $1142(25.9)$ & $459(40.2)$ & $0.69(0.59-0.80)^{*}$ \\
\hline \multicolumn{4}{|l|}{ SES (poverty) } \\
\hline 1st (low SES) & $989(22.4)$ & $457(46.2)$ & Referent \\
\hline 2nd & $1070(24.2)$ & $504(47.1)$ & $1.07(0.89-1.29)$ \\
\hline $3 \mathrm{rd}$ & $1139(25.8)$ & $549(48.2)$ & $1.13(0.93-1.37)$ \\
\hline 4th (high SES) & $1220(27.6)$ & $567(46.5)$ & $1.10(0.89-1.35)$ \\
\hline \multicolumn{4}{|l|}{ Region } \\
\hline Midwest & $566(12.8)$ & 247 (43.6) & Referent \\
\hline North east & $1000(22.6)$ & $443(44.3)$ & $0.95(0.76-1.20)$ \\
\hline South & $1035(23.4)$ & $533(51.5)$ & $1.31(1.05-1.64)^{*}$ \\
\hline
\end{tabular}


Table 4 continued

\begin{tabular}{|c|c|c|c|}
\hline Characteristics & $\begin{array}{l}\text { mCRC patients who } \\
\text { received first-line } \\
\text { treatment, } \\
N(\text { column \%) }\end{array}$ & $\begin{array}{l}\text { mCRC patients who } \\
\text { received first-line with } \\
\text { targeted biologic, } \\
N(\text { row } \%)\end{array}$ & $\begin{array}{l}\text { Receipt of first-line } \\
\text { targeted biologic, } \\
\text { multivariable } \\
\text { OR }(95 \% \mathrm{CI})\end{array}$ \\
\hline West & $2969(67.2)$ & $854(47.0)$ & $1.06(0.86-1.32)$ \\
\hline \multicolumn{4}{|l|}{ Urban/rural } \\
\hline Less urban/rural & $477(10.8)$ & $210(44.0)$ & Referent \\
\hline Urban & $268(6.1)$ & $126(47.0)$ & $1.24(0.90-1.71)$ \\
\hline Metro & $3672(83.1)$ & $1741(47.4)$ & $1.29(1.03-1.61)^{*}$ \\
\hline \multicolumn{4}{|l|}{ Year of diagnosis } \\
\hline 2004 & 731 (16.6) & $112(15.3)$ & Referent \\
\hline 2005 & $696(15.8)$ & $368(52.9)$ & $6.55(5.09-8.44)^{*}$ \\
\hline 2006 & $780(17.7)$ & $447(57.3)$ & $7.62(5.93-9.78) *$ \\
\hline 2007 & $722(16.3)$ & $391(54.2)$ & $6.70(5.20-8.62)^{*}$ \\
\hline 2008 & $752(17.0)$ & $376(50.0)$ & $5.80(4.51-7.45)^{*}$ \\
\hline 2009 & 737 (16.7) & $383(52.0)$ & $6.21(4.83-7.98)^{*}$ \\
\hline
\end{tabular}

$C I$ confidence interval, $m C R C$ metastatic colorectal cancer, $O R$ odds ratio, SES socio-economic status

* Significant at $\alpha=0.05$

Treatment sequences administered to patients, along with durations, are shown in Table 3. The most common treatment sequence was first-line oxaliplatin or irinotecan followed by second-line oxaliplatin or irinotecan + bevacizumab followed by a third-line targeted biologic (OIOIB-TB). This sequence was given to nearly $11 \%$ of patients, with a median of 86 days first-line, 160 days second-line, and 146 days third-line treatment (Table 3). The second most common sequence ( $8 \%$ ) was first-line oxaliplatin or irinotecan + bevacizumab (median 195 days) followed by second-line oxaliplatin or irinotecan + bevacizumab (median 143 days) followed by a third-line targeted biologic (median 132 days; OIB-OIBTB). Other common sequences (Table 3) received by patients were first-line oxaliplatin or irinotecan followed by second-line oxaliplatin or irinotecan + bevacizumab (OIOIB) and first-line oxaliplatin or irinotecan + bevacizumab followed by second-line oxaliplatin or irinotecan + bevacizumab (OIB-OIB). For sequences OI-OIB and OIB-OIB, no third-line treatment was observed. Sequences with bevacizumab in first line were observed to be administered for a relatively longer duration of time than sequences without bevacizumab in first line (Table 3).

\subsection{Factors Associated with Receipt of Targeted Biologic and Treatment Sequences}

Characteristics of patients receiving targeted biologics at first-line therapy, along with logistic regression results, are shown in Table 4. Of patients who received first-line therapy, $47 \%$ also received a targeted biologic (Table 4). As compared with patients aged 65-69 years, patients aged 75-79 or 80-84 years were less likely to receive targeted biologics (Table 4). Patients with a comorbidity score of 1 and with metastatic rectal cancer were also less likely to receive a targeted biologic at first line. Patients residing in the South as well as in metropolitan areas were relatively more likely to receive targeted biologics and, as expected, utilization of targeted biologics was higher among patients diagnosed in the years 2005-2009 than among those diagnosed in 2004 (Table 4).

Table 5 shows the univariate comparison of characteristics of patients who received the commonly administered targeted biologic-based treatment sequences using the chisquared statistic. In the univariate analysis between treatment sequences, statistically significant differences were only observed with regards to comorbidity score, other metastasis, and year of diagnosis (Table 5). Factors associated with commonly administered treatment sequences, assessed using multinomial logistic regression with treatment sequence (four categories) as the dependent variable and OI-OIB as the reference category, are presented in Table 6. Patients aged 75-79 years were significantly less likely to receive three-line treatment sequences, i.e., OIBOIB-TB and OI-OIB-TB, than an OI-OIB treatment sequence (Table 6). Female mCRC patients were observed to be 0.37 times less likely to receive an OIB-OIB-TB treatment sequence, and patients with a comorbidity score of 1 (vs. 0) were less likely to receive OIB-OIB-TB, OIOIB-TB, and OIB-OIB treatment sequences (Table 6). 
Table 5 Characteristics of metastatic colorectal cancer patients by commonly administered treatment sequences

\begin{tabular}{|c|c|c|c|c|c|}
\hline \multirow[t]{2}{*}{ Characteristics } & \multicolumn{5}{|l|}{ Treatment sequences } \\
\hline & OI-OIB-TB $(N=275)$ & OIB-OIB-TB $(N=199)$ & OI-OIB $(N=178)$ & OIB-OIB $(N=169)$ & $P$ value \\
\hline Age (years) & & & & & 0.0600 \\
\hline $65-69$ & $108(39.3)$ & $81(40.7)$ & $56(31.5)$ & $56(33.1)$ & \\
\hline $70-74$ & $84(30.6)$ & $72(36.2)$ & $57(32.0)$ & $49(29.0)$ & \\
\hline $75-79$ & $56(20.4)$ & $32(16.1)$ & $47(26.4)$ & $38(22.5)$ & \\
\hline$\geq 80$ & $27(9.8)$ & $14(7.0)$ & $18(10.1)$ & $26(15.4)$ & \\
\hline Race/ethnicity & & & & & 0.4093 \\
\hline Caucasian & $235(85.5)$ & $173(86.9)$ & $144(80.9)$ & $142(84.0)$ & \\
\hline Other & $40(14.6)$ & $26(13.1)$ & $34(19.1)$ & $27(16.0)$ & \\
\hline Sex & & & & & 0.1751 \\
\hline Male & $161(58.6)$ & $113(56.8)$ & $86(48.3)$ & $91(53.9)$ & \\
\hline Female & $114(41.5)$ & $86(43.2)$ & $92(51.7)$ & $78(46.2)$ & \\
\hline Marital status & & & & & 0.2983 \\
\hline Married & $189(68.7)$ & $125(62.8)$ & $114(64.0)$ & $102(60.4)$ & \\
\hline Unmarried/unknown & $86(31.3)$ & $74(37.2)$ & $64(36.0)$ & $67(39.6)$ & \\
\hline Tumor grade & & & & & 0.7064 \\
\hline Well/moderately differentiated & $176(64.0)$ & $121(60.8)$ & $105(59.0)$ & $107(63.3)$ & \\
\hline Poorly/undifferentiated/unknown & $99(36.0)$ & $78(39.2)$ & $73(41.0)$ & $62(36.7)$ & \\
\hline Comorbidity scores & & & & & $0.0129 *$ \\
\hline 0 & $173(62.9)$ & $123(61.8)$ & $84(47.2)$ & 97 (57.4) & \\
\hline 1 & $71(25.8)$ & 49 (24.6) & $69(38.8)$ & $44(26.0)$ & \\
\hline$\geq 2$ & $31(11.3)$ & $27(13.6)$ & $25(14.0)$ & $28(16.6)$ & \\
\hline \multicolumn{6}{|l|}{ Metastasis } \\
\hline Liver & $206(74.9)$ & 143 (71.9) & $126(70.8)$ & $128(75.7)$ & 0.6458 \\
\hline Lung & $41(14.9)$ & $31(15.6)$ & $22(12.4)$ & $26(15.4)$ & 0.8079 \\
\hline Abdomen & $57(20.7)$ & 37 (18.6) & $31(17.4)$ & $25(14.8)$ & 0.4608 \\
\hline Other & 43 (15.6) & $19(9.6)$ & $34(19.1)$ & $18(10.7)$ & $0.0248 *$ \\
\hline Unknown & $37(13.5)$ & $29(14.6)$ & $31(17.4)$ & $24(14.2)$ & 0.7010 \\
\hline Cancer site & & & & & 0.0829 \\
\hline Colon & 194 (70.6) & 155 (77.9) & $128(71.9)$ & 135 (79.9) & \\
\hline Rectal & $81(29.5)$ & $44(22.1)$ & $50(28.1)$ & $34(20.1)$ & \\
\hline SES (poverty) & & & & & 0.2732 \\
\hline 1st (low SES) & $62(22.6)$ & 41 (20.6) & $34(19.1)$ & $41(24.3)$ & \\
\hline $2^{\text {nd }}$ & $56(20.4)$ & $53(26.6)$ & $48(27.0)$ & 37 (21.9) & \\
\hline $3 \mathrm{rd}$ & $69(25.1)$ & $53(26.6)$ & $43(24.2)$ & $54(32.0)$ & \\
\hline 4th (high SES) & $88(32.0)$ & $52(26.1)$ & $53(29.8)$ & 37 (21.9) & \\
\hline Region & & & & & 0.0915 \\
\hline Midwest & $31(11.3)$ & $18(9.1)$ & $20(11.2)$ & $15(8.9)$ & \\
\hline North east & $55(20.0)$ & $41(20.6)$ & $38(21.4)$ & $36(21.3)$ & \\
\hline South & $45(16.4)$ & 45 (22.6) & $49(27.5)$ & $48(28.4)$ & \\
\hline West & $144(52.4)$ & $95(47.7)$ & $71(39.9)$ & 70 (41.4) & \\
\hline Urban/rural & & & & & 0.1894 \\
\hline Less urban/rural & $22(8.0)$ & $18(9.1)$ & $19(10.7)$ & $24(14.2)$ & \\
\hline Metro/urban & $253(92.0)$ & $181(91.0)$ & 159 (89.3) & $145(85.8)$ & \\
\hline Year of diagnosis & & & & & $<0.0001 *$ \\
\hline 2004-2005 & 109 (39.6) & $52(26.1)$ & $56(31.5)$ & $40(23.7)$ & \\
\hline 2006-2007 & $90(32.7)$ & $95(47.7)$ & $48(27.0)$ & $56(33.1)$ & \\
\hline 2008-2009 & 76 (27.6) & $52(26.1)$ & 74 (41.6) & $73(43.2)$ & \\
\hline
\end{tabular}

Data are presented as $n(\%)$ unless otherwise indicated

OI-OIB first-line oxaliplatin or irinotecan followed by second-line oxaliplatin or irinotecan + bevacizumab, OIB-OIB first-line oxaliplatin or irinotecan + bevacizumab followed by second-line oxaliplatin or irinotecan + bevacizumab, OI-OIB-TB OI-OIB followed by a third-line targeted biologic, $O I B-O I B-T B$ OIB-OIB followed by a third-line targeted biologic, $S E S$ socio-economic status

* Significant at $\alpha=0.05$ 
Table 6 Multinomial logistic regression for factors associated with receipt of commonly administered treatment sequences

\begin{tabular}{|c|c|c|c|}
\hline Factors & OI-OIB-TB & OIB-OIB-TB & OIB-OIB \\
\hline \multicolumn{4}{|l|}{ Age (years) } \\
\hline $65-69$ & Referent & Referent & Referent \\
\hline $70-74$ & $0.87(0.52-1.45)$ & $0.77(0.47-1.25)$ & $0.89(0.52-1.54)$ \\
\hline $75-79$ & $0.43(0.24-0.77)^{*}$ & $0.59(0.35-1.00)^{*}$ & $0.78(0.43-1.41)$ \\
\hline $80+$ & $0.45(0.20-1.01)$ & $0.73(0.36-1.50)$ & $1.36(0.65-2.85)$ \\
\hline \multicolumn{4}{|l|}{ Race/ethnicity } \\
\hline Caucasian & Referent & Referent & Referent \\
\hline Other & $0.54(0.29-1.00)$ & $0.63(0.37-1.10)$ & $0.74(0.41-1.36)$ \\
\hline \multicolumn{4}{|l|}{ Sex } \\
\hline Male & Referent & Referent & Referent \\
\hline Female & $0.63(0.41-0.99)^{*}$ & $0.67(0.44-1.00)$ & $0.76(0.48-1.19)$ \\
\hline \multicolumn{4}{|l|}{ Marital status } \\
\hline Married & Referent & Referent & Referent \\
\hline Unmarried/unknown & $1.29(0.81-2.05)$ & $0.94(0.61-1.45)$ & $1.29(0.81-2.06)$ \\
\hline \multicolumn{4}{|l|}{ Tumor grade } \\
\hline Well/moderately differentiated & Referent & Referent & Referent \\
\hline Poorly/undifferentiated/unknown & $0.94(0.6-1.46)$ & $0.83(0.55-1.26)$ & $0.91(0.57-1.44)$ \\
\hline \multicolumn{4}{|l|}{ Comorbidity scores } \\
\hline 0 & Referent & Referent & Referent \\
\hline 1 & $0.45(0.28-0.72)^{*}$ & $0.52(0.34-0.81)^{*}$ & $0.51(0.31-0.84)^{*}$ \\
\hline$\geq 2$ & $0.61(0.32-1.18)$ & $0.57(0.31-1.06)$ & $0.92(0.48-1.74)$ \\
\hline \multicolumn{4}{|l|}{ Metastasis (yes vs. no) } \\
\hline Liver & $0.77(0.36-1.66)$ & $1.16(0.58-2.36)$ & $1.00(0.45-2.25)$ \\
\hline Lung & $1.36(0.72-2.57)$ & $1.21(0.67-2.18)$ & $1.38(0.72-2.65)$ \\
\hline Abdomen & $1.03(0.53-1.98)$ & $1.28(0.71-2.30)$ & $0.84(0.43-1.67)$ \\
\hline Other & $0.39(0.20-0.75)^{*}$ & $0.83(0.47-1.44)$ & $0.50(0.26-0.98) *$ \\
\hline Unknown & $0.56(0.21-1.47)$ & $0.87(0.36-2.15)$ & $0.65(0.23-1.80)$ \\
\hline \multicolumn{4}{|l|}{ Cancer site } \\
\hline Colon & Referent & Referent & Referent \\
\hline Rectal & $0.62(0.38-1.03)$ & $1.01(0.65-1.59)$ & $0.59(0.35-1.00)^{*}$ \\
\hline \multicolumn{4}{|l|}{ SES (poverty) } \\
\hline 1st (low SES) & Referent & Referent & Referent \\
\hline 2nd & $0.92(0.48-1.77)$ & $0.55(0.30-1.02)$ & $0.70(0.36-1.37)$ \\
\hline $3 \mathrm{rd}$ & $0.88(0.44-1.76)$ & $0.65(0.35-1.23)$ & $1.09(0.55-2.16)$ \\
\hline 4th (high SES) & $0.69(0.34-1.41)$ & $0.69(0.36-1.31)$ & $0.58(0.28-1.21)$ \\
\hline \multicolumn{4}{|l|}{ Region } \\
\hline Midwest & Referent & Referent & Referent \\
\hline North east & $1.38(0.60-3.19)$ & $0.87(0.41-1.84)$ & $1.59(0.66-3.79)$ \\
\hline South & $0.88(0.39-2.02)$ & $0.51(0.24-1.07)$ & $1.22(0.53-2.84)$ \\
\hline West & $1.43(0.66-3.10)$ & $1.17(0.59-2.32)$ & $1.37(0.61-3.09)$ \\
\hline \multicolumn{4}{|l|}{ Urban/rural } \\
\hline Less urban/rural & Referent & Referent & Referent \\
\hline Metro/urban & $1.25(0.57-2.74)$ & $1.25(0.59-2.61)$ & $0.76(0.36-1.62)$ \\
\hline \multicolumn{4}{|l|}{ Year of diagnosis } \\
\hline 2004-2005 & Referent & Referent & Referent \\
\hline 2006-2007 & $2.55(1.48-4.40)$ & $1.17(0.71-1.93)$ & $1.75(0.97-3.14)$ \\
\hline 2008-2009 & $0.84(0.49-1.45)$ & $0.59(0.36-0.94)^{*}$ & $1.50(0.87-2.57)$ \\
\hline
\end{tabular}

Data are presented as odds ratio (95\% confidence interval)

$O I B$-OIB first-line oxaliplatin or irinotecan + bevacizumab followed by second-line oxaliplatin or irinotecan + bevacizumab, OI-OIB-TB OI-OIB followed by a third-line targeted biologic, OIB-OIB-TB OIB-OIB followed by a third-line targeted biologic, SES socio-economic status

* Significant at $\alpha=0.05$ 
Metastatic rectal cancer patients were also less likely to receive an OIB-OIB treatment sequence.

\section{Discussion}

Important advances in treatments for mCRC patients over the last decade have provided clinicians with a multitude of treatment options. The addition of oxaliplatin or irinotecan to 5-FU/LV increased the median survival up to 19.5 months as compared with 14.8 months with 5-FU/LV alone $[48,49]$. Moreover, the availability of targeted biologics such as bevacizumab has been found to increase the overall survival to as high as 25.5 months [50]. Thus, irinotecan- or oxaliplatin-based chemotherapy regimens have been recommended, and the addition of bevacizumab has been considered a reasonable option [16, 17]. Sequencing of these chemotherapies and targeted biologics is equally important, as treatments received during the first few months of the diagnosis are critical [7, 51, 52], but correct sequencing of treatments could be challenging, and evidence of current utilization patterns may be informative $[16,17]$. We used community-based SEER-Medicare linked data to identify the treatment patterns, sequences, and associated factors for mCRC patients diagnosed from 2004 to 2009.

FOLFOX- or oxaliplatin-based regimens were most frequently administered to mCRC patients as their first-line treatment, which is consistent with previous findings among relatively younger patients $[6,11,38]$. Bikov et al. [53] found 5-FU-based treatment to be the most common therapy and oxaliplatin-based therapy as the second most common treatment; however, their analyses only included elderly metastatic colon cancer patients diagnosed until 2007 [53], and prescribing patterns may have changed in the subsequent years. The observation that FOLFOX- or oxaliplatin-based regimens are preferred as first-line treatment is consistent with their relatively better toxicity profile as compared with FOLFIRI- or irinotecan-based regimens $[50,54,55]$. However, a recent systematic review concluded that first-line oxaliplatin- or irinotecan-based regimens are equally efficacious for mCRC patients [16], and the efficacy and safety of these treatments for elderly patients has been found to be comparable to that in younger patients [15]. Nearly half of the patients had a targeted biologic as a part of their first-line treatment, with a significant increase in patients receiving a targeted biologic in the last decade as shown by the results of our study and that by Abrams et al. [38]. As the evidence supporting the survival benefit of targeted biologics for elderly patients becomes more widespread, elderly mCRC patients may often receive targeted biologics during first-line treatment. Alternatively, we also found that patients in older age groups, 75-79 and 80-84 years, and patients with higher comorbidity scores had a lower likelihood of receiving a targeted biologic in first line, which reflects concerns with regards to prevalence of more comorbidities, cardiovascular, and cerebrovascular toxicities, and less access to specialist care in these older patients $[14,56,57]$.

Consistent with previous findings, FOLFIRI- or irinotecan-based regimens were relatively more common in second-line treatment, and cetuximab + irinotecan was the most common regimen at the third line $[11,53]$. Treatment sequencing showed mCRC patients receiving treatments (first to third line) in various sequences. The two most common sequences consisted of patients receiving three lines of treatment, with the difference being the receipt of bevacizumab in the first line. Bevacizumab was commonly observed to be administered as second-line treatment in combination with chemotherapy among patients who had previously been treated with bevacizumab in the first line. Previous studies by Abrams et al. [38] and Hess et al. [11] found similar results, but treatment with bevacizumab in second line following progression with bevacizumab in first line was not recommended during the time of the study. However, based on some recent studies, it has now been included in the National Comprehensive Cancer Network guidelines [58-60]. Our results show that mCRC patients receive treatments in sequences that may not necessarily be recommended or clinically shown to have survival benefit. While clinical trials $[19,20]$ are underway to definitively examine the comparative efficacy of different treatment sequences, comparative-effectiveness studies using community-level data may provide evidence to better inform clinicians. Additionally, as patients may receive a treatment continuum to prolong their survival, the overall cost to treat mCRC patients would increase considerably and thereby necessitate economic evaluation of treatment sequences.

Study results should be interpreted in light of the following limitations. First, identification of lines of treatment administered was limited to the first three lines. Since, $80-90 \%$ of mCRC patients receive a maximum of three lines of treatment $[11,61]$, this limitation should not substantially reduce the applicability of our findings. Second, only drugs that require administration by a healthcare provider were considered for our analysis, and orally administered drugs (e.g., capecitabine) were not included because Medicare part D data were not available. A previous study by Hess et al. [11] found that capecitabine was administered to $8.9 \%$ at first line, $4.9 \%$ at second line, and $6.9 \%$ at third line. Third, we only included patients with a gap between treatment lines of less than 1 year. However, additional analyses including these patients showed results similar to our primary analyses (Tables 2 and 3 in the ESM). Fourth, the factors assessed were 
limited to patient and tumor characteristics available from the SEER-Medicare dataset and did not include patient or physician preferences, which are known to influence treatment receipt. Finally, findings of the study are only generalizable to $\mathrm{mCRC}$ patients aged $\geq 65$ years who are not enrolled in Medicare Part C plans.

\section{Conclusion}

Based on the study results, we observed that elderly mCRC patients receive a treatment continuum with multiple drugs administered across various lines of treatment. As recommended and similar to studies in younger populations, oxaliplatin- or irinotecan-based regimens were the most common chemotherapies, with bevacizumab the most common targeted biologic administered. Treatment sequencing studies using real-world data among overall and elderly mCRC populations are limited, and future studies should evaluate the utilization of treatment sequences using other national data sources. Additionally, studies assessing the comparative and cost effectiveness of the most common treatment sequences identified should be conducted to provide evidence-based recommendations for clinicians and policy makers.

Acknowledgments The authors acknowledge the efforts of the National Cancer Institute; Center for Medicare and Medicaid Services; Information Management Services, Inc.; and the SEER Program tumor registries in the creation of this database. The interpretation and reporting of these data are the sole responsibilities of the authors.

\section{Compliance with Ethical Standards}

Ethical approval The study was determined as exempt by the Committee for the Protection of Human Subjects at the University of Texas Health Science Centre at Houston.

Conflicts of interest Rohan C. Parikh, Xianglin L. Du, Robert O. Morgan and David R. Lairson have no conflicts of interest that are directly relevant to the content of this study

Funding This study was supported in part by a grant from the Agency for Healthcare Research and Quality (R01-HS018956) and in part by a grant from the Cancer Prevention and Research Institute of Texas (RP130051). The funding agencies had no involvement in the study design; in the collection, analysis, and interpretation of data; in the writing of the manuscript; or in the decision to submit the manuscript for publication.

Open Access This article is distributed under the terms of the Creative Commons Attribution-NonCommercial 4.0 International License (http://creativecommons.org/licenses/by-nc/4.0/), which permits any noncommercial use, distribution, and reproduction in any medium, provided you give appropriate credit to the original author(s) and the source, provide a link to the Creative Commons license, and indicate if changes were made.

\section{References}

1. Siegel R, DeSantis C, Jemal A. Colorectal cancer statistics, 2014. CA Cancer J Clin. 2014;64(2):104-17.

2. American Cancer Society. Colorectal Cancer Facts \& Figures 2011-2013. Atlanta: American Cancer Society; 2011.

3. Siegel RL, Miller KD, Jemal A. Cancer statistics, 2015. CA Cancer J Clin. 2015;65(1):5-29.

4. Chu E. An update on the current and emerging targeted agents in metastatic colorectal cancer. Clin Colorectal Cancer. 2012;11(1):1-13.

5. SEER Stat Fact Sheets: Colon and rectum cancer [online]. 2015. Available from: http://seer.cancer.gov/statfacts/html/colorect. html. Accessed 15 Dec 2015.

6. Seal BS, Sullivan SD, Ramsey S, Shermock KM, Ren J, Kreilick $\mathrm{C}$, et al. Medical costs associated with use of systemic therapy in adults with colorectal cancer. J Manag Care Pharm. 2013;19(6):461-7.

7. Viele CS. Metastatic colorectal cancer: the treatment continuum. Seminars in Oncology Nursing. Philadelphia (PA): Elsevier; 2007.

8. National Comprehensive Cancer Network. Clinical practice guidelines in oncology: colon cancer; 2014. http://www.nccn.org. Accessed 15 May 2015.

9. National Comprehensive Cancer Network. Clinical practice guidelines in oncology: rectal cancer; 2014. http://www.nccn.org. Accessed 15 May 2015.

10. Board RE, Valle JW. Metastatic colorectal cancer. Drugs. 2007;67(13):1851-67.

11. Hess GP, Wang PF, Quach D, Barber B, Zhao Z. Systemic therapy for metastatic colorectal cancer: patterns of chemotherapy and biologic therapy use in us medical oncology practice. J Oncol Pract. 2010;6(6):301-7.

12. Meyerhardt JA, Mayer RJ. Systemic therapy for colorectal cancer. N Engl J Med. 2005;352(5):476-87.

13. Goldberg RM. Therapy for metastatic colorectal cancer. Oncologist. 2006;11(9):981-7.

14. Sanoff HK, Goldberg RM. How we treat metastatic colon cancer in older adults. J Geriatr Oncol. 2013;4(4):295-301.

15. Papamichael D, Audisio R, Horiot JC, Glimelius B, Sastre J, Mitry E, et al. Treatment of the elderly colorectal cancer patient: SIOG expert recommendations. Ann Oncol. 2009;20(1):5-16.

16. Bekaii-Saab T, Wu C. Seeing the forest through the trees: a systematic review of the safety and efficacy of combination chemotherapies used in the treatment of metastatic colorectal cancer. Crit Rev Oncol. 2014;91(1):9-34.

17. Lee JJ, Chu E. Sequencing of anti-angiogenic agents in the treatment of metastatic colorectal cancer. Clin Colorectal Cancer. 2014;13(3):135-44.

18. Benson $\mathrm{AB}$ 3rd. Epidemiology, disease progression, and economic burden of colorectal cancer. J Manag Care Pharm. 2007;13(6 Suppl C):S5-18.

19. Multi-line therapy trial in unresectable metastatic colorectal cancer (STRATEGIC-1) [online]. 2014. Available from: http:// clinicaltrials.gov/show/NCT01910610. Accessed 15 Dec 2015.

20. Sequential treatment strategy for metastatic colorectal cancer (ITACa) [online]. 2014. Available from: http://clinicaltrials.gov/ show/NCT01878422. Accessed 15 Dec 2015.

21. Biganzoli L, Goldhirsch A, Straehle C, Castiglione-Gertsch M, Therasse P, Aapro M, et al. Adjuvant chemotherapy in elderly patients with breast cancer: a survey of the Breast International Group (BIG). Ann Oncol. 2004;15(2):207-10.

22. Neugut AI, Matasar M, Wang X, McBride R, Jacobson JS, Tsai WY, et al. Duration of adjuvant chemotherapy for colon cancer and survival among the elderly. J Clin Oncol. 2006;24(15):2368-75. 
23. Potosky AL, Harlan LC, Kaplan RS, Johnson KA, Lynch CF. Age, sex, and racial differences in the use of standard adjuvant therapy for colorectal cancer. J Clin Oncol. 2002;20(5):1192-202.

24. Schrag D, Cramer LD, Bach PB, Begg CB. Age and adjuvant chemotherapy use after surgery for stage III colon cancer. J Natl Cancer Inst. 2001;93(11):850-7.

25. Sundararajan V, Mitra N, Jacobson JS, Grann VR, Heitjan DF, Neugut AI. Survival associated with 5-fluorouracil-based adjuvant chemotherapy among elderly patients with node-positive colon cancer. Ann Intern Med. 2002;136(5):349-57.

26. Warren JL, Klabunde CN, Schrag D, Bach PB, Riley GF. Overview of the SEER-Medicare data: content, research applications, and generalizability to the United States elderly population. Med Care. 2002;40(8):IV,3-IV-18.

27. Overview of the SEER program [Internet].; 2015. Available from: http://seer.cancer.gov/about/overview.html. Accessed 15 Dec 2015.

28. Engels EA, Pfeiffer RM, Ricker W, Wheeler W, Parsons R, Warren JL. Use of surveillance, epidemiology, and end resultsmedicare data to conduct case-control studies of cancer among the US elderly. Am J Epidemiol. 2011;174(7):860-70.

29. Warren JL, Harlan LC, Fahey A, Virnig BA, Freeman JL, Klabunde $\mathrm{CN}$, et al. Utility of the SEER-Medicare data to identify chemotherapy use. Med Care. 2002;40(8 Suppl):IV,55-61.

30. Zippin C, Lum D, Hankey BF. Completeness of hospital cancer case reporting from the SEER Program of the National Cancer Institute. Cancer. 2006;76(11):2343-50.

31. Du XL, Parikh RC, Lairson DR, Giordano SH, Cen P. Comparative effectiveness of platinum-based chemotherapy versus taxane and other regimens for ovarian cancer. Med Oncol. 2013;30(1):1-14.

32. Lairson DR, Parikh RC, Cormier JN, Chan W, Du XL. Costutility analysis of chemotherapy regimens in elderly patients with stage III colon cancer. Pharmacoeconomics. 2014;32(10):1005-13.

33. Thrall MM, Gray HJ, Symons RG, Weiss NS, Flum DR, Goff BA. Trends in treatment of advanced epithelial ovarian cancer in the Medicare population. Gynecol Oncol. 2011;122(1):100-6.

34. Thrall MM, Gray HJ, Symons RG, Weiss NS, Flum DR, Goff BA. Neoadjuvant chemotherapy in the Medicare cohort with advanced ovarian cancer. Gynecol Oncol. 2011;123(3):461-466.

35. Gruschkus SK, Lairson D, Dunn JK, Risser J, Du XL. Comparative effectiveness of white blood cell growth factors on neutropenia, infection, and survival in older people with nonhodgkin's lymphoma treated with chemotherapy. J Am Geriatr Soc. 2010;58(10):1885-95.

36. Du XL, Osborne C, Goodwin JS. Population-based assessment of hospitalizations for toxicity from chemotherapy in older women with breast cancer. J Clin Oncol. 2002;20(24):4636-42.

37. North East SAS Users Group (NESUG). 2011. http://www. lexjansen.com/nesug/nesug11/ph/ph07.pdf. Accessed 6 June 2015.

38. Abrams TA, Meyer G, Schrag D, Meyerhardt JA, Moloney J, Fuchs CS. Chemotherapy usage patterns in a US-wide cohort of patients with metastatic colorectal cancer. J Natl Cancer Inst. 2014;106(2):djt371.

39. Seal BS, Sullivan SD, Ramsey SD, Shermock KM, Ren J, Kreilick C, et al. Systemic therapy for colorectal cancer: patterns of chemotherapy and biologic therapy use in nationally representative US claims database. BioDrugs. 2014;28(2):229-236.

40. Charlson ME, Pompei P, Ales KL, MacKenzie CR. A new method of classifying prognostic comorbidity in longitudinal studies: development and validation. J Chronic Dis. 1987;40(5):373-83.

41. Deyo RA, Cherkin DC, Ciol MA. Adapting a clinical comorbidity index for use with ICD-9-CM administrative databases. J Clin Epidemiol. 1992;45(6):613-9.
42. Klabunde CN, Potosky AL, Legler JM, Warren JL. Development of a comorbidity index using physician claims data. J Clin Epidemiol. 2000;53(12):1258-67.

43. Chawla N, Yabroff KR, Mariotto A, McNeel TS, Schrag D, Warren JL. Limited validity of diagnosis codes in Medicare claims for identifying cancer metastases and inferring stage. Ann Epidemiol. 2014;24(9):666,672. e2.

44. Lund JL, Sturmer T, Harlan LC, Sanoff HK, Sandler RS, Brookhart MA, et al. Identifying specific chemotherapeutic agents in Medicare data: a validation study. Med Care. 2013;51(5):e27-34.

45. Hosmer Jr DW, Lemeshow S. Applied logistic regression. London: Wiley; 2004.

46. Davidoff AJ, Tang M, Seal B, Edelman MJ. Chemotherapy and survival benefit in elderly patients with advanced non-small-cell lung cancer. J Clin Oncol. 2010;28(13):2191-7.

47. Schonberg MA, Marcantonio ER, Li D, Silliman RA, Ngo L, McCarthy EP. Breast cancer among the oldest old: tumor characteristics, treatment choices, and survival. J Clin Oncol. 2010;28(12):2038-45.

48. Saltz LB, Cox JV, Blanke C, Rosen LS, Fehrenbacher L, Moore $\mathrm{MJ}$, et al. Irinotecan plus fluorouracil and leucovorin for metastatic colorectal cancer. N Engl J Med. 2000;343(13):905-14.

49. Goldberg RM, Sargent DJ, Morton RF, Fuchs CS, Ramanathan RK, Williamson SK, et al. A randomized controlled trial of fluorouracil plus leucovorin, irinotecan, and oxaliplatin combinations in patients with previously untreated metastatic colorectal cancer. J Clin Oncol. 2004;22(1):23-30.

50. Bendell JC, Bekaii-Saab TS, Cohn AL, Hurwitz HI, Kozloff M, Tezcan $\mathrm{H}$, et al. Treatment patterns and clinical outcomes in patients with metastatic colorectal cancer initially treated with FOLFOX-bevacizumab or FOLFIRI-bevacizumab: results from ARIES, a bevacizumab observational cohort study. Oncologist. 2012;17(12):1486-95.

51. Lange A, Prenzler A, Frank M, Kirstein M, Vogel A, von der Schulenburg J. A systematic review of cost-effectiveness of monoclonal antibodies for metastatic colorectal cancer. Eur $\mathbf{J}$ Cancer. 2014;50(1):40-9.

52. Cartwright TH. Treatment decisions after diagnosis of metastatic colorectal cancer. Clin Colorectal Cancer. 2012;11(3):155-66.

53. Bikov KA, Mullins CD, Seal B, Onukwugha E, Hanna N. Algorithm for identifying chemotherapy/biological regimens for metastatic colon cancer in SEER-Medicare. Med Care. 2015;53(8):e58-64.

54. Colucci G, Gebbia V, Paoletti G, Giuliani F, Caruso M, Gebbia $\mathrm{N}$, et al. Phase III randomized trial of FOLFIRI versus FOLFOX4 in the treatment of advanced colorectal cancer: a multicenter study of the Gruppo Oncologico Dell'Italia Meridionale. J Clin Oncol. 2005;23(22):4866-75.

55. Pasetto LM, Jirillo A, Iadicicco G, Rossi E, Paris MK, Monfardini S FOLFOX versus FOLFIRI: a comparison of regimens in the treatment of colorectal cancer metastases. Anticancer Res. 2005;25(1B):563-76.

56. Kozloff MF, Berlin J, Flynn PJ, Kabbinavar F, Ashby M, Dong $\mathrm{W}$, et al. Clinical outcomes in elderly patients with metastatic colorectal cancer receiving bevacizumab and chemotherapy: results from the BRiTE observational cohort study. Oncology. 2010;78(5-6):329-39.

57. Saltz LB, Clarke S, Diaz-Rubio E, Scheithauer W, Figer A, Wong $\mathrm{R}$, et al. Bevacizumab in combination with oxaliplatin-based chemotherapy as first-line therapy in metastatic colorectal cancer: a randomized phase III study. J Clin Oncol. 2008;26(12):2013-9.

58. Arnold D, Andre T, Bennouna J, Sastre J, Osterlund PJ, Greil R, et al. Bevacizumab (BEV) plus chemotherapy (CT) continued beyond first progression in patients with metastatic colorectal cancer (mCRC) previously treated with BEV plus CT: Results of a randomized phase III intergroup study (TML study). ASCO Annual meeting proceedings; 2012. 
59. Cartwright TH, Yim YM, Yu E, Chung H, Halm M, Forsyth M. Survival outcomes of bevacizumab beyond progression in metastatic colorectal cancer patients treated in US community oncology. Clin colorectal cancer. 2012;11(4):238-46.

60. Benson AB,3rd, Bekaii-Saab T, Chan E, Chen YJ, Choti MA, Cooper HS, et al. Metastatic colon cancer, version 3.2013: featured updates to the NCCN Guidelines. J Natl Compr Canc Netw. 2013;11(2):141,52 (quiz 152).

61. Chastek B, Kulakodlu M, Valluri S, Seal B. Impact of metastatic colorectal cancer stage and number of treatment courses on patient healthcare costs and utilization. Postgrad Med. 2013;125(2):73-82. 